\title{
First in-core gamma spectroscopy experiments in a zero power reactor
}

\author{
Oskari Pakari ${ }^{1}$, Vincent Lamirand ${ }^{1,2}$, Tom Mager ${ }^{1}$, Axel Laureau ${ }^{1}$, and Pavel Frajtag ${ }^{1}$, and Andreas Pautz ${ }^{1,2}$ \\ ${ }^{1}$ Laboratory for Reactor Physics and Systems Behaviour \\ Ecole polytechnique fédérale de Lausanne \\ ${ }^{2}$ Laboratory for Reactor Physics and Thermal-Hydraulics \\ Nuclear Energy and Safety Division \\ Paul Scherrer Institut, Villigen, Switzerland
}

Corresponding author: oskari.pakari@gmail.com

\begin{abstract}
-
Gamma rays in nuclear reactors, arising either from nuclear reactions or decay processes, significantly contribute to the heating and dose of the reactor components. Zero power research reactors offer the possibility to measure gamma rays in a purely neutronic environment, allowing for validation experiments of dose estimates, computed spectra, and prompt to delayed gamma ratios. The resulting data can contribute to models, code validation and photo atomic/nuclear data evaluation. To date, most experiments have relied on flux measurements using TLDs, ionization chambers, or spectrometers set in low flux areas. The CROCUS reactor allows for flexible detector placement in and around the core, and has recently been outfitted with gamma detection capabilities to fulfill the need for in-core gamma spectroscopy, as opposed to flux. In this paper we report on the experiments and accompanying simulations of gamma spectrum measurements inside a zero power reactor core, CROCUS. It is a two-zone, uranium-fueled light water moderated facility operated by the Laboratory for Reactor Physics and Systems Behaviour (LRS) at the Swiss Federal Institute of Technology Lausanne (EPFL). Herein we also introduce, in detail, the new LEAF system: A Large Energy-resolving detection Array for Fission gammas. It consists of an array of four detectors - two large $\varnothing 127 \times 254 \mathrm{~mm}$ Bismuth Germanate (BGO) and two smaller $\varnothing 12 \times 50 \mathrm{~mm}$ Cerium Bromide $\left(\mathrm{CeBr}_{3}\right)$ scintillators. We describe the calibration and characterization of LEAF followed by first in-core measurements of gamma ray spectra in a zero power reactor at different sub-critical and critical states, and different locations. The spectra are then compared to code results, namely MCNP6.2 pulse height tallies. We were able to distinguish prompt processes and delayed peaks from decay databases. We present thus experimental data from hitherto inaccessible core regions. We provide the data as validation means for codes that attempt to model these processes for energies up to $10 \mathrm{MeV}$. We finally draw conclusions and discuss the future uses of LEAF. The results indicate the possibility of isotope tracking and burn-up validation.
\end{abstract}

Index Terms-Gamma spectroscopy, Zero power research reactor.

\section{INTRODUCTION}

G AMMA radiation as a by-product of fission and radioactive decay is a major component of a nuclear reactor's radiation field. It contributes to the heating of structures, the degradation of materials, and the overall dose rate around a reactor [1]. The characteristics of a reactor's gamma field is thus important for operation and safety. Accurate predictions are desired in operational planning, in the design of experiments in research facilities, and in the inception of new reactors [2]. The sources of gamma radiation in nuclear reactors include the prompt fission process, delayed radioactive decay, and nuclear capture reactions. Experiments often target the individual processes to single out their effect; examples range from spent fuel spectroscopy [3], [4] to fission product decay spectra and direct fission gamma spectra [5], [6].

Quantitative experiments on the spectra and fluxes of gamma radiation have become of interest with the introduction of coupled photon and neutron transport options in common Monte Carlo codes, such as Serpent [7] and MCNP [8] - recently compared for the specific case of CROCUS in [9]. Measurements of nuclear reactor gamma ray fields have included spectroscopy outside of the reactor shielding [10], and in-core measurements of fluxes using ionization chambers [11]-[13]. A recent focus has been the determination of delayed gamma contributions measured with mixed neutron-gamma flux measurements in research reactors [14], [15]. To the knowledge of the authors, no direct measurements of in-core gamma spectra have hitherto been performed. The CROCUS research reactor offers a flexible zero power environment for these types of experiments. To date, TLD measurements of the gamma field of CROCUS have been conducted in the context of activation and far field flux analysis. Furthermore, combined neutron/gamma measurements using a sCVD detector [16] have added to the radiation field flux characterization. With a variety of other experimental programs [17] that so far only exploited neutron detection [18], [19], we extended the capabilities of CROCUS to state of the art gamma detection capabilities.

This is the intent of the LEAF system: A Large Energyresolving detection Array for Fission gammas to directly measure gamma ray spectra in CROCUS. We hereby aim at providing measurement data in previously unexplored param- 


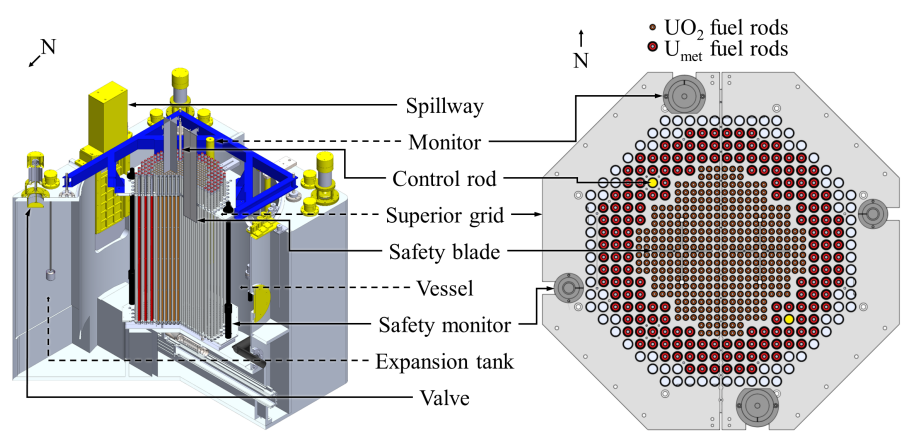

Fig. 1. Schematic isometric view of the CROCUS reactor (left), and top view of the core configuration (right).

eter regions, namely in-core and high energy $(>2 \mathrm{MeV})$ spectroscopy. In this paper we present the characterization and first measurements of said system - notably the first measurement of gamma ray spectra from an in-core location. We facilitate a possible code validation by providing a detailed overview of the geometry. In Section II we introduce the experimental details: the CROCUS reactor, a detailed description of LEAF and the energy calibration of all detectors, as well as the experimental setup used for spectroscopy. In Section III we show the results of measurements using LEAF in CROCUS - namely in-core and ex-core spectra - and compare the results to MCNP6.2 simulations and thereafter discuss their implications.

\section{Methods AND MATERIALS}

\section{A. The Experimental Reactor CROCUS}

A full description of the core can be found in the International Reactor Physics Experiments Handbook (IRPhE) [20], [21]. The CROCUS reactor is a two-zone, uranium-fueled, $\mathrm{H}_{2} \mathrm{O}$-moderated critical assembly operated by the Laboratory for Reactor Physics and Systems Behaviour (LRS) at the Swiss Federal Institute of Technology Lausanne (EPFL). It is a zero power reactor, with a maximum power of $100 \mathrm{~W}$. The core is approximately cylindrical in shape with a diameter of about $58 \mathrm{~cm}$ and a height of $100 \mathrm{~cm}$. Two different kinds of fuel rods make up the reactor core of CROCUS (see Fig. 1). The central zone is loaded with $336 \mathrm{UO}_{2}$ fuel rods (1.806 wt.\%enriched), set in a square lattice with a pitch of $1.837 \mathrm{~cm}$. The peripheral zone is loaded with up to 176 thicker, $\mathrm{U}_{m e t}$ fuel rods (0.947 wt.\%-enriched) with a pitch of $2.917 \mathrm{~cm}$, also in a square lattice. All fuel rods are cladded with aluminum and are maintained in a vertical position by the upper grid and lower grid plates, spaced $100 \mathrm{~cm}$ apart. In the current COLIBRI configuration [22]-[25], both grid plates incorporate a cadmium layer with a thickness of $1 \mathrm{~mm}$ to limit axial thermal leakage to surrounding structures. This is also the configuration for the herein presented results. The active fuel length starts at the top surface of the lower cadmium layer and extends to $100 \mathrm{~cm}$. The core is located in an aluminum water tank, its diameter is $130 \mathrm{~cm}$ and thickness is $1.2 \mathrm{~cm}$. Demineralized light water is used as moderator and reflector. Reactivity is nominally controlled by a variation of the water level using a spillway with an accuracy of $0.1 \mathrm{~mm}$ (equivalent to $0.4 \mathrm{pcm}$ ) and optionally by means of two control rods containing naturally enriched boron carbide $\left(\mathrm{B}_{4} \mathrm{C}\right)$ sintered pellets located in diagonal symmetry within the outer fuel zone.

\section{B. The LEAF system: Overview and Calibration}

We specifically intend to expand the capabilities of CROCUS by a dedicated gamma detection system. Scintillators were hereby the final choice when optimizing for radiation hardness, flexible use, price, and nonetheless high efficiency when compared to semiconductor based detectors. In total, an array of four detectors was acquired from Scionix Holland [26] to allow for symmetric placement of the system in and around the core (see Figure 7). The high voltage supply and photomultiplier tube (PMT) signal amplification was handled by the Mirion Technologies DSA-LX [27] (here designated 419 and 420 as per serial number) that theoretically allow for $\sim \mathrm{MHz}$ count rates to be treated. In the following we introduce the respective detectors in detail and present their characteristics and calibration.

\section{1) Cerium Bromide $\left(\mathrm{CeBr}_{3}\right)$ detectors}

When the two control rods are removed, their guide tubes are a prominent location for in-core measurements, e.g. used for delayed gamma fraction estimation [28], intrinsic gamma noise [29], and induced neutron/gamma noise measurements [22], [23]. Cerium(III) Bromide $\left(\mathrm{CeBr}_{3}\right)$, as a comparatively new scintillation material [30], [31], was chosen as the most suitable material for a high photon flux zone with a comparatively fast decay time of $20 \mathrm{~ns}$ at a density of $5.2 \mathrm{~g} / \mathrm{cm}^{3}$ [32]. The radiation hardness was estimated to be well sufficient for use in CROCUS [9], [33]. In order to fit the guide tubes, the cylindrical crystals measure $13 \mathrm{~mm}$ in diameter and $15 \mathrm{~mm}$ in length, see Figure 2. Both detectors use a Hamamatsu Type R12421 PMT.

\section{2) Bismuth Germanate (BGO) detectors}

To measure gamma rays in ex-core locations, specifically photons with energies above $2 \mathrm{MeV}$, a material with high photon stopping efficiency was desired. Bismuth Germanate (BGO) is a well established scintillator in various detection applications [34], [35] with slower decay and lower light yield than the $\mathrm{CeBr}_{3}$ [36], but with a higher density of $7.13 \mathrm{~g} / \mathrm{cm}^{3}$ and lower price per volume. To meet an absorption efficiency of above $95 \%$ for $10 \mathrm{MeV}$ photons the two acquired cylindrical crystals are therefore $127 \mathrm{~mm}$ in diameter. The height was constrained by weight $(25 \mathrm{~kg})$ and price to $250 \mathrm{~mm}$. Both are equipped with Photonis 5" Type XP4578 PMTs, see Figure 3.

3) Amplification settings and energy calibration of LEAF

For both detectors and all experiments presented herein the DSA-LX's settings were as follows:

- Rise time of $0.2 \mu \mathrm{s}, 0.0 \mu \mathrm{s}$ flat top.

- $-610 \mathrm{~V}$ and $-1260 \mathrm{~V}$ of $\mathrm{HV}$ for the $\mathrm{CeBr}_{3}$ and BGO PMTs, respectively.

- Lower level discrimination at $0.5 \%$ of the maximum channel $\left(2^{14}\right)$.

- Coarse gain of 6.4 .

All four detectors were calibrated using standard Eu-152 sources - the resulting spectra are displayed in Figures 4 


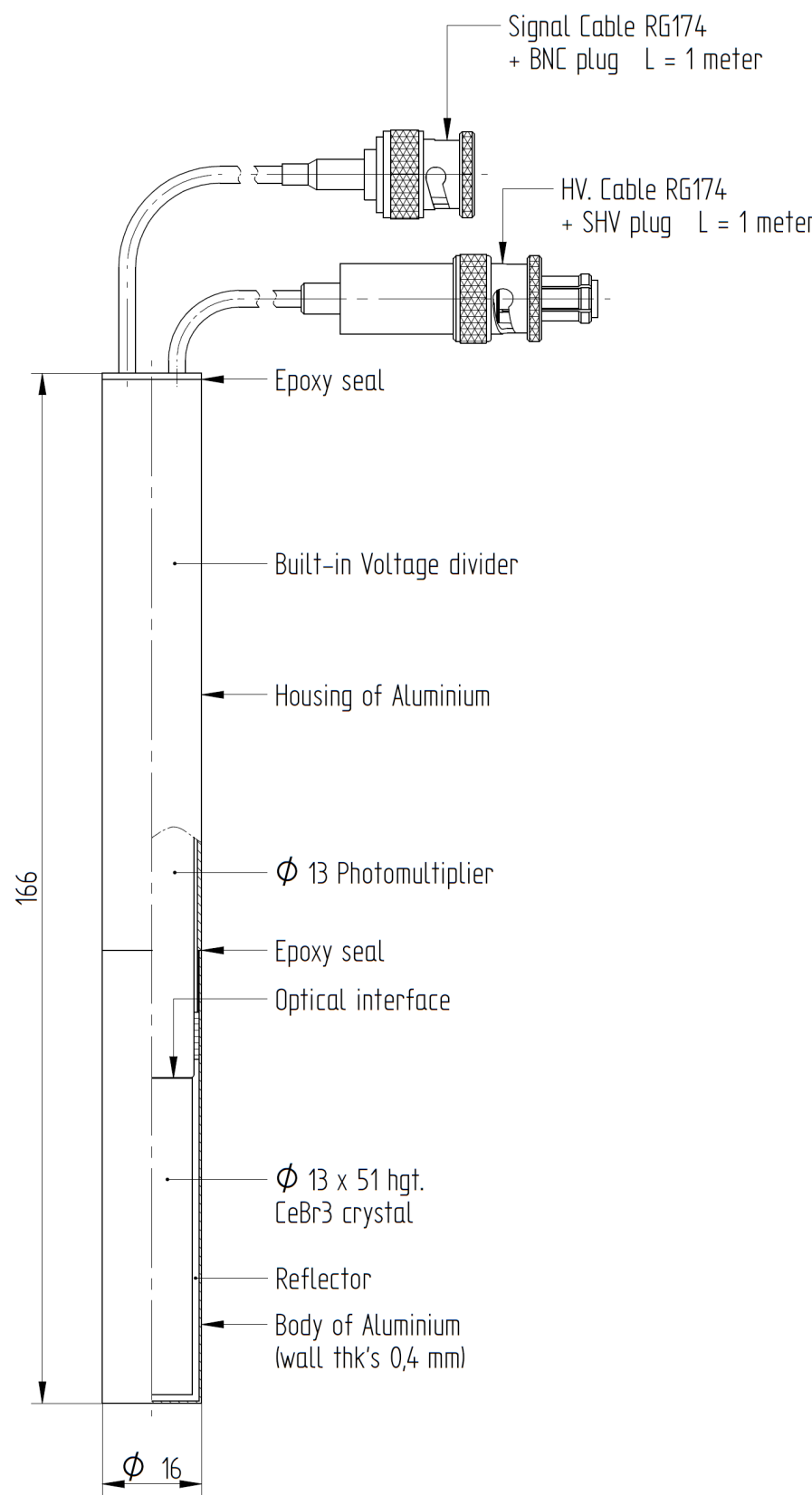

Fig. 2. Technical drawing of the $\mathrm{CeBr}_{3}$ detector as provided by Scionix, used to create models for Monte Carlo transport codes. The PMT is a Hamamatsu Type R12421.

and 5. The BGO, due to its comparatively higher full width at half maximum (FWHM), required additionally a Co-60 measurement to aid in the peak distinction. Each identified peak was fitted with a Gaussian to find the mean value used as input for the calibration. The final calibrations for all detectors is linear with an $\mathrm{R}^{2}>0.99$. The calibration was purposefully undertaken using only the first quarter of channels as higher energy events were expected. We also examined the FWHM (see Figure 6) of all detectors with energy and found them to be comparable to other crystals in literature, e.g. [37].
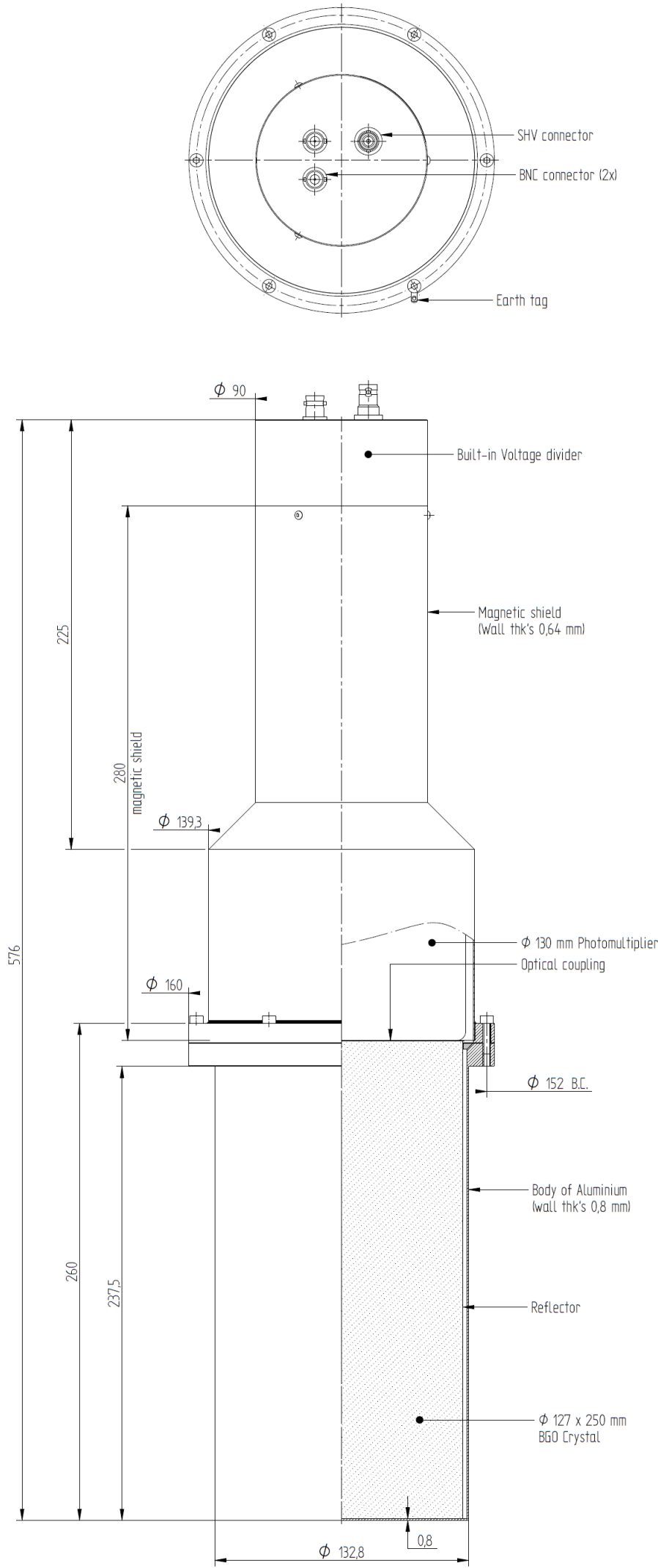

Fig. 3. Technical drawing of the BGO detector as provided by Scionix, used to create models for Monte Carlo transport codes. The PMT is a Photonis 5" Type XP4578 PMT. 

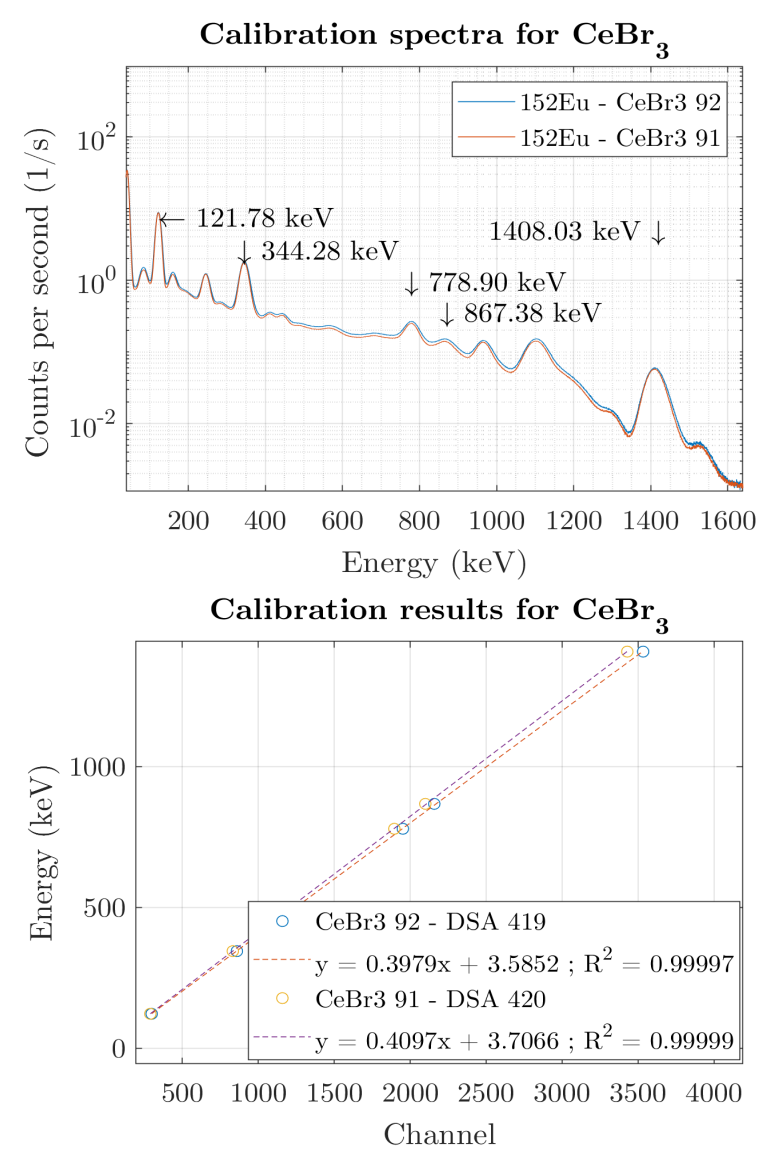

Fig. 4. Top: Calibration gamma spectra acquired with the $\mathrm{CeBr}_{3}$ detectors Bottom: Resulting linear calibration fits after extracting the peak center values via Gaussian fitting of each peak.

\section{Experimental setup: LEAF in CROCUS}

The $\mathrm{CeBr}_{3}$ detectors were placed at mid core height in the control rod tubes, while the BGO detectors were set just outside of the main vessel, as displayed in Figure 7, also at mid core height. The acquisition layout with the electronics is sketched in Figure 8. CROCUS in its shutdown state contains no water in the vessel. In the start-up procedure water is filled step-wise until $800 \mathrm{~mm}$, after which the operator has control over it. For the experiments in this work we chose a set of subcritical and critical states to use LEAF in a variety of reactor states. The configurations, water levels and estimated fission powers are listed in Table I. The system shows - consistently across the detectors - an excellent counting response up to $0.2 \mathrm{MHz}$ which in this setup corresponded to about $100 \mathrm{~mW}$ of power [38]. This is about two orders of magnitude better than the in-house developed neutron noise detection system based on regular gamma spectrometry electronics [39].

D. Simulation of gamma spectra in CROCUS using MCNP6.2

MCNP6.2 offers the possibility to simulate coupled neutron photon transport with pulse height estimation tallies for detectors. These simulations herein include only prompt
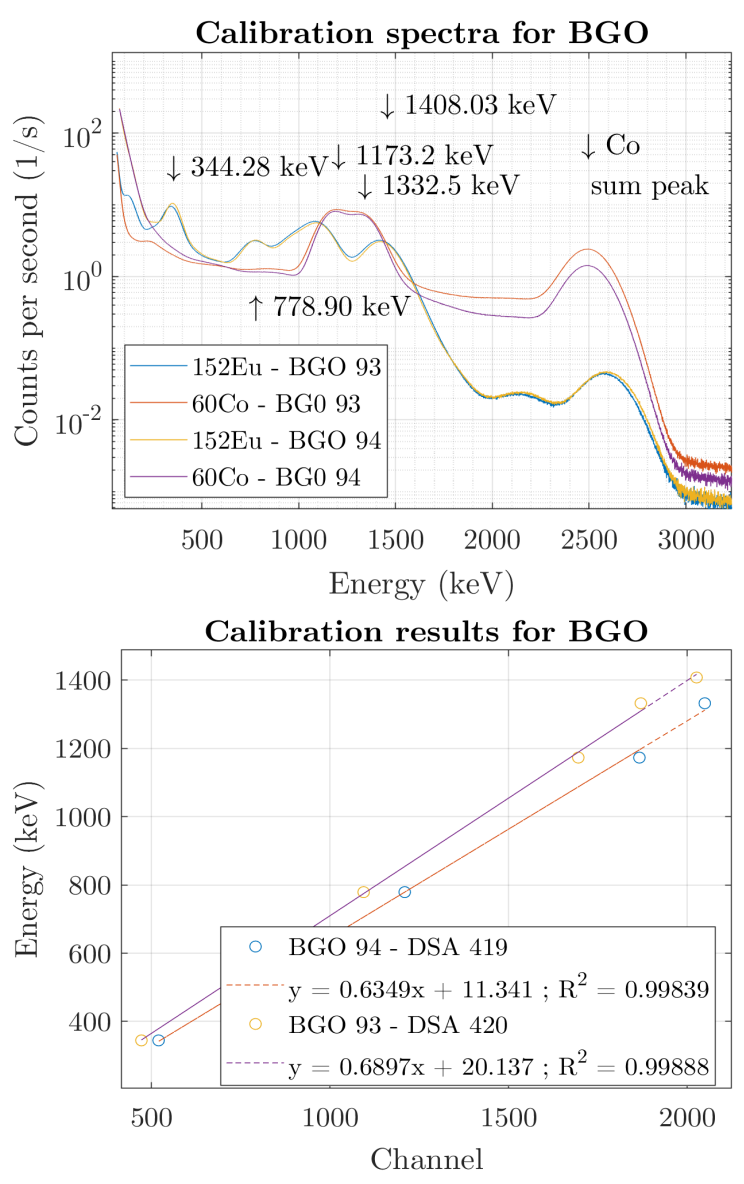

Fig. 5. Top: Calibration gamma spectra acquired with the BGO detectors. Bottom: Resulting linear calibration fits after extracting the peak center values via Gaussian fitting of each peak.

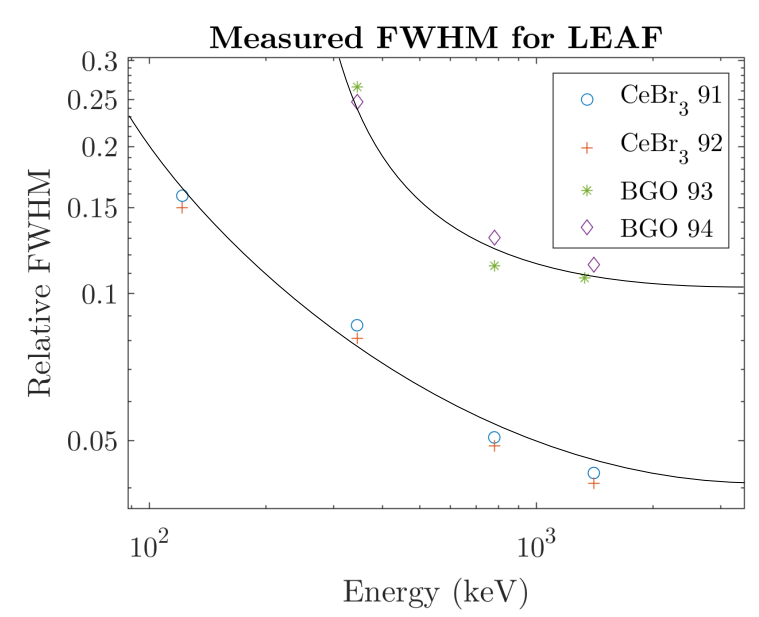

Fig. 6. FWHM for the LEAF detectors in dependence of energy, determined with a standard Eu-152 source. The fitted lines are for illustrative purposes.

processes, i.e. fission and, if available in the nuclear data used (ENDF/B-7.1), (n, $\gamma)$ photons. In this work we compare the results qualitatively to the energy deposition tallies without taking smearing into account, e.g. due to the scintillation process and the electronics . 


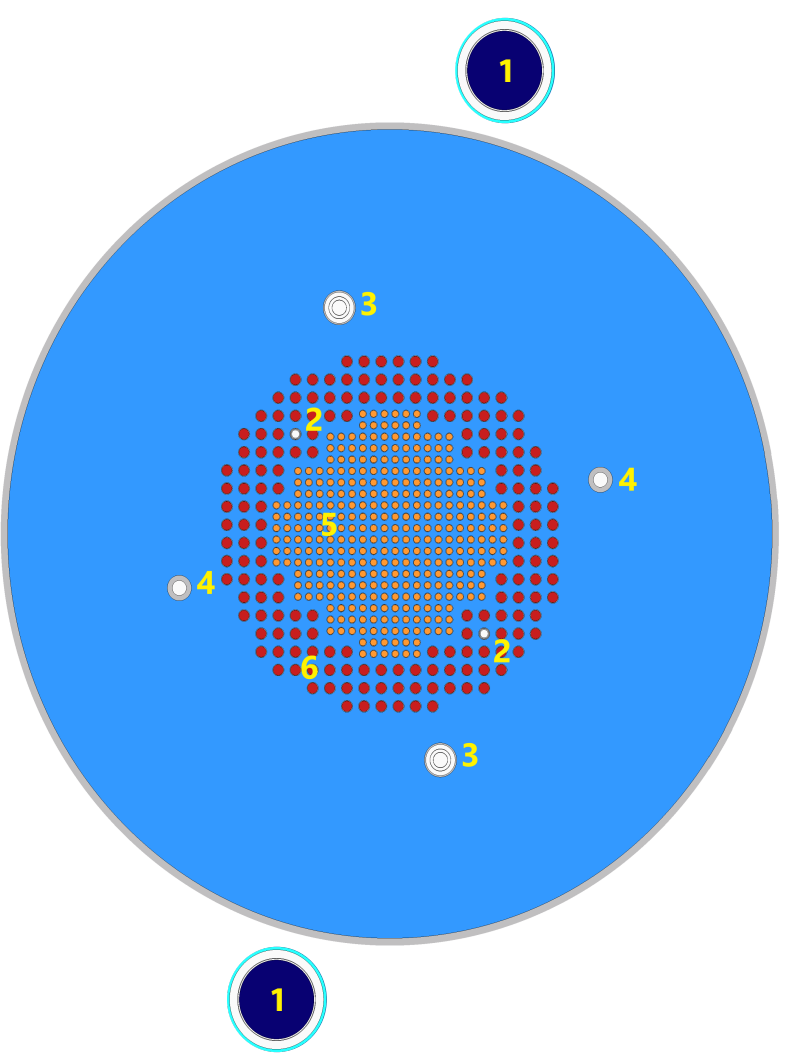

Fig. 7. Top view of the Serpent 2 model of CROCUS with LEAF at midcore height. 1) BGO detector, 2) $\mathrm{CeBr}_{3}$ detector, 3) Compensated ionization chamber, 4) Power monitors, 5) $\mathrm{UO}_{2}$ fuel lattice, 6) $\mathrm{U}_{\text {met }}$ fuel lattice.

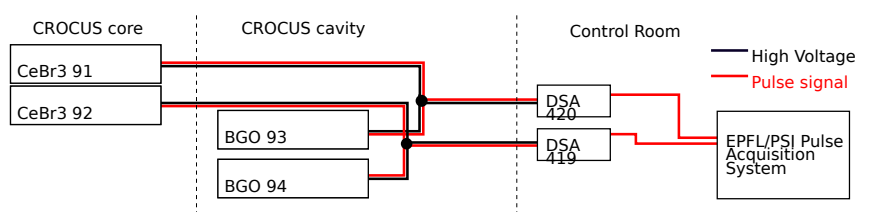

Fig. 8. Schematic of the acquisition layout for the gamma spectroscopy experiment in CROCUS using LEAF.

TABLE I

OVERVIEW OF THE CROCUS CONFIGURATIONS USED FOR THE GAMMA SPECTROSCOPY EXPERIMENTS.

\begin{tabular}{lcc}
\hline \hline Configuration & $\begin{array}{c}\text { Water level } \\
\mathrm{mm}\end{array}$ & $\begin{array}{c}\text { Fission power estimate [38] } \\
\mathrm{mW}\end{array}$ \\
\hline Shutdown & Empty & 0 \\
H3 & 500.0 & 1.2 \\
Sub-critical: & 800.0 & 3.3 \\
& 850.0 & 3.9 \\
& 900.0 & 6.1 \\
Critical: & 925.0 & 9.4 \\
\hline \hline
\end{tabular}

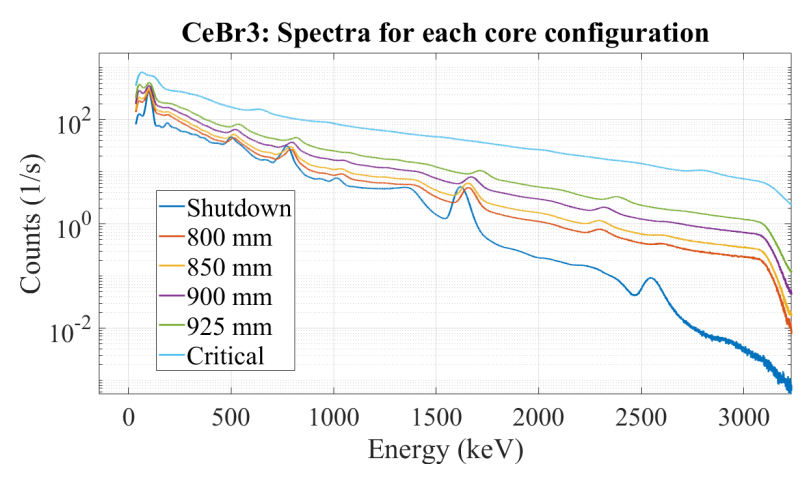

Fig. 9. Measured in-core gamma spectra using a $\mathrm{CeBr}_{3}$ detector in the control rod position for sub-critical and critical configurations.

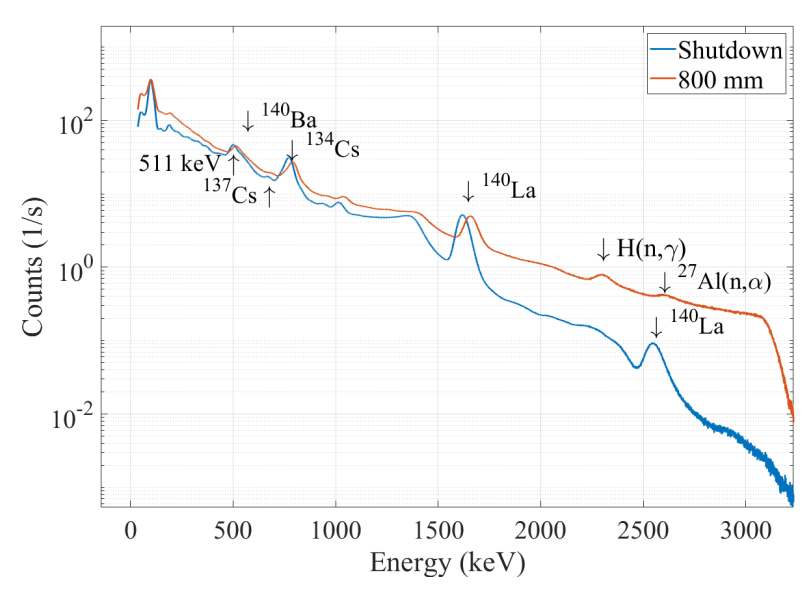

Fig. 10. Measured in-core gamma spectra using a $\mathrm{CeBr}_{3}$ detector in the control rod position for $800 \mathrm{~mm}$ and the shutdown state. Preliminary identified peaks are indicated in the plot using the data from Table II.

\section{RESULTS}

\section{A. $\mathrm{CeBr}_{3}$ : First recorded in-core gamma spectra}

In Figure 9 we display $\mathrm{CeBr}_{3}$ spectra acquired at different reactor configurations, ranging from shutdown to criticality. We most strikingly observe the expected exponential shape of the spectrum from fission with several resolved peaks from specific decays on top. We note a gain shift in the spectrum to higher energies with higher count rate, an effect often found in PMTs at high event rates [40]. The spectra notably all exhibit a cut-off at around $3.3 \mathrm{MeV}$. Using NIST standard data on photon mass attenuation lengths [41] and the density mentioned before, we find that photons of this energy have a mean free path of $3.8 \mathrm{~cm}$ - a value consistent with the detector geometry.

\section{B. BGO: Coarse ex-vessel spectra up to $10 \mathrm{MeV}$}

In Figure 11 we display BGO spectra acquired at $500 \mathrm{~mm}$ water level with and without the PuBe start-up neutron source of CROCUS below the core [42]. At higher water levels the spectra showed only an exponential shape induced by fission with no visually identifiable peaks, similar to the spectrum of the $\mathrm{CeBr}_{3}$ at criticality. By comparing spectra with and 


\section{BGO: Subcritical source experiment}

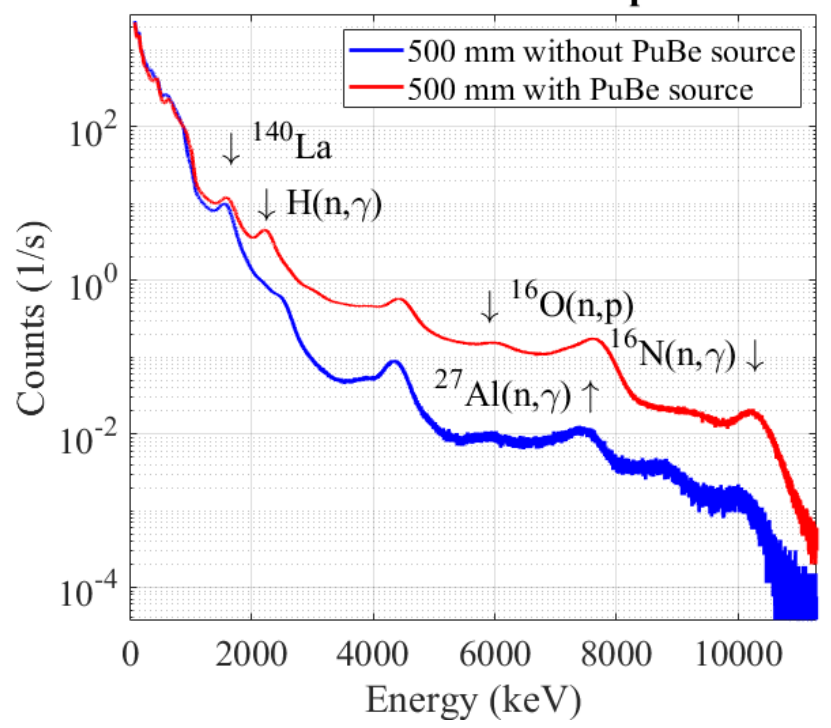

Fig. 11. Measured gamma spectra using a BGO detector in the core vesse periphery for the $\mathrm{H} 3$ sub-critical state, with and without the start-up neutron source below the core. Identified peaks are indicated in the plot.

TABLE II

OVERVIEW OF EMISSIONS USED FOR PEAK IDENTIFICATION. ENERGIES ROUNDED TO THE CLOSEST KEV. THE HALF LIFE IS INDICATED FOR BETA DELAYED GAMMA EMISSIONS.

\begin{tabular}{llll}
\hline \hline Reaction & Energy $(\mathrm{keV})$ & Half life & Reference \\
\hline Annihilation & 511 & prompt & {$[43]$} \\
${ }^{140} \mathrm{Ba}$ & 537 & $12.7 \mathrm{~d}$ & {$[44]$} \\
${ }^{137} \mathrm{Cs}$ & 662 & $30.5 \mathrm{a}$ & {$[44]$} \\
${ }^{134} \mathrm{Cs}$ & 795 & $2.1 \mathrm{a}$ & {$[44]$} \\
${ }^{140} \mathrm{La}$ & 816 & $1.7 \mathrm{~d}$ & {$[44]$} \\
${ }^{140} \mathrm{La}$ & 1596 & $1.7 \mathrm{~d}$ & {$[44]$} \\
${ }^{27} \mathrm{Al}(\mathrm{n}, \gamma){ }^{28} \mathrm{Si}$ & 1779 & $2.3 \mathrm{~min}$ & {$[45]$} \\
${ }^{1} \mathrm{H}(\mathrm{n}, \gamma)$ & 2223 & prompt & {$[43]$} \\
${ }^{140} \mathrm{La}$ & 2521 & $1.68 \mathrm{~d}$ & {$[44]$} \\
${ }^{27} \mathrm{Al}(\mathrm{n}, \alpha)^{24} \mathrm{Na}$ & 2754 & $15.0 \mathrm{~h}$ & {$[44]$} \\
${ }^{16} \mathrm{O}(\mathrm{n}, \mathrm{p})^{16} \mathrm{~N}$ & 6140 & $7.1 \mathrm{~s}$ & {$[43]$} \\
${ }^{27} \mathrm{Al}(\mathrm{n}, \gamma)$ & 7724 & prompt & {$[46]$} \\
${ }^{14} \mathrm{~N}(\mathrm{n}, \gamma)$ & 10829 & prompt & {$[43]$} \\
\hline \hline
\end{tabular}

without source we attempted to distinguish neutron induced prompt lines from longer lived activation and fission products. Indeed, we find a general increase in spectral baseline due to fission gammas - and also a new line e.g. at around $2.2 \mathrm{MeV}$ and $6.1 \mathrm{MeV}$. In the next section we discuss the gamma line identification and implications.

\section{Discussion}

Peak identification given the here presented data is challenging. To approach this problem we herein use two methods: Firstly, by accessing various decay and neutron capture databases we chose a list of likely causes based on expert judgement, listed in Table II. Secondly, we simulated the prompt gamma transport in MCNP6.2 in the respective detector volumes and overlaid the F8 pulse height tally over the experimental data - as shown in Figures 12 and 13 for the $\mathrm{CeBr}_{3}$ and $\mathrm{BGO}$, respectively. Note that the spectra are

\section{$\mathrm{CeBr}_{3}$ : Experiment vs. Simulation}

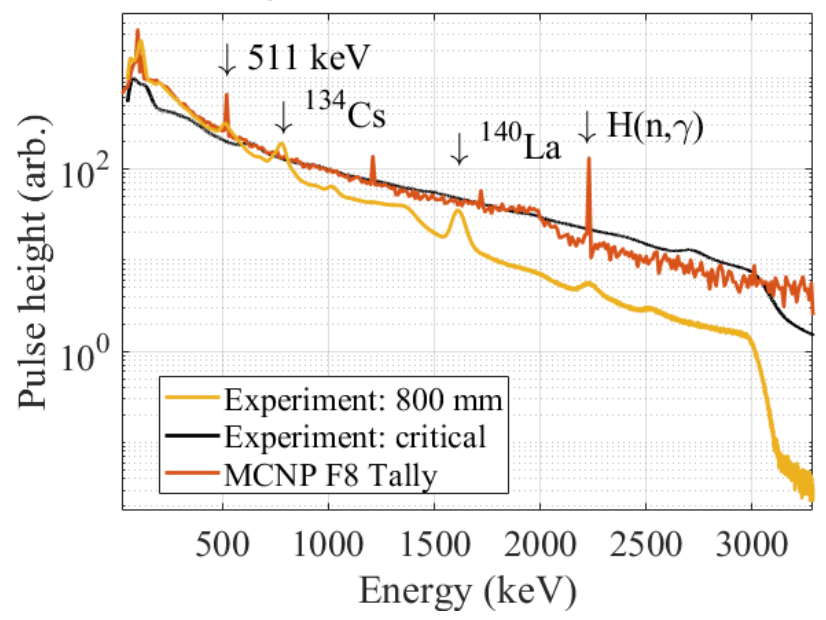

Fig. 12. Comparison of experimental in-core gamma spectra using a $\mathrm{CeBr}_{3}$ detector in the control rod position to the MCNP pulse height tally (F8). Preliminary identified peaks are indicated in the plot.

arbitrarily normalized to allow for qualitative comparison of the shapes.

We most notably identify across both in-core and ex-vessel spectra:

- Several unidentified lines below $500 \mathrm{keV}$ that could correspond to a range of fission products but we refrain for attempting an identification in this work.

- Long lived fission products relevant for waste and fallout quantification [47] such as ${ }^{137} \mathrm{Cs},{ }^{134} \mathrm{Cs},{ }^{140} \mathrm{La}$, and ${ }^{140} \mathrm{Ba}$.

- Radiative neutron capture and activation, mostly from $\mathrm{H}(n, \gamma)$ and potentially the surrounding aluminum structures. In the high energy spectra of the BGO we furthermore observe the $6.1 \mathrm{MeV}$ and $10.8 \mathrm{MeV}{ }^{16} \mathrm{~N}$ decay lines.

We emphasize here that the identified peaks using the outlined methods (MCNP correlation and databases) is preliminary. Future work will focus on quantifying the decay behavior of the peaks to establish a more solid causal link. An unresolved inconsistency is for example the line at $\sim 4.4 \mathrm{MeV}$ as seen in the BGO spectra in Figures 11 and 13. According to MCNP, it is exactly twice the energy of the hydrogen capture gamma, yet it is found in both spectra with and without the PuBe neutron source. We nonetheless can state that the MCNP simulations qualitatively agree with the measurements for other expected prompt processes, such as annihilation and capture in hydrogen, and disagree when it comes to fission product decay such as ${ }^{140} \mathrm{La}$. This highlights the need and opportunity for validation taking delayed gammas and signal smearing into account.

The results are overall promising: fission products could be directly measured in-core, opening the possibility to track their production on-line as well as validate burnup calculations of CROCUS [48]. 


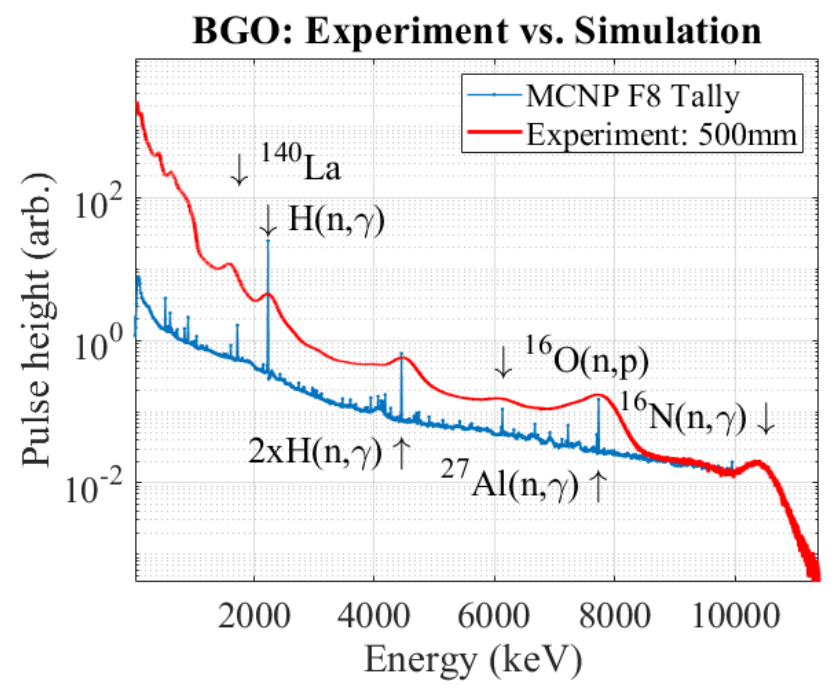

Fig. 13. Comparison of experimental BGO gamma spectrum to the MCNP pulse height tally (F8). Preliminary identified peaks are indicated in the plot.

\section{CONCLUSION}

In this work we present, to the best of the authors' knowledge, for the first time gamma spectra from a central region of a nuclear reactor. By using a modern scintillator material $\left(\mathrm{CeBr}_{3}\right)$ with comparatively low FWHM, we were able to distinguish peaks above the fission spectrum. We also present the measurement of ex-core gamma ray spectra up to $10 \mathrm{MeV}$ using BGO detectors that also show several peaks.

We introduced the new gamma spectroscopy system for the CROCUS zero power reactor called LEAF. It consists of four scintillation based detectors, two $\mathrm{CeBr}_{3}$ and two large BGO crystals, each with different specifications allowing for a wide range of experimental reactor applications. We presented detailed geometry, characteristics and calibration to allow for explicit modeling and ultimately aspire to provide benchmark quality measurements for validation purposes.

The measurements of in-core gamma spectra were presented notably at different sub-critical and critical states of CROCUS. We also presented sub-critical measurements with and without start-up neutron source to highlight the difference with and without fission and other neutron induced interactions. Additionally, the experiments are compared to pulse height tallies produced with MCNP6.2. The experiments qualitatively agree with the simulations with regards to prompt spectrum shape and preliminary $(n, \gamma)$ line identification. Resolved peaks of delayed processes not modeled in aforementioned codes were tentatively identified using databases, most notably the fission products ${ }^{134} \mathrm{Cs}$ and ${ }^{140} \mathrm{La}$, verifying the need for more sophisticated simulations taking delayed processes into account [49].

In the future, in addition to reactor gamma spectroscopy, LEAF will be used for reactor perturbation studies [50], reactor noise [51] in the gamma regime as well as further investigations into gamma ray spectra in- and ex-core with emphasis on delayed process identification and quantification.

\section{REFERENCES}

[1] A.-C. Colombier, H. Amharrak, D. Fourmentel, S. Ravaux, D. Régnier, O. Gueton, J.-P. Hudelot, and M. Lemaire, "Nuclear data production, calculation and measurement: a global overview of the gamma heating issue," EPJ Web of Conferences, vol. 42, p. 04001, 2013.

[2] G. Rimpault, D. Bernard, D. Blanchet, C. Vaglio-Gaudard, S. Ravaux, and A. Santamarina, "Needs of accurate prompt and delayed $\gamma$-spectrum and multiplicity for nuclear reactor designs," Physics Procedia, vol. 31, pp. 3-12, 2012.

[3] D. Beddingfield and F. Cecil, "Identification of fissile materials from fission product gamma-ray spectra," Nuclear Instruments and Methods in Physics Research Section A: Accelerators, Spectrometers, Detectors and Associated Equipment, vol. 417, no. 2-3, pp. 405-412, nov 1998.

[4] C. Willman, A. Håkansson, O. Osifo, A. Bäcklin, and S. J. Svärd, "Nondestructive assay of spent nuclear fuel with gamma-ray spectroscopy," Annals of Nuclear Energy, vol. 33, no. 5, pp. 427-438, mar 2006.

[5] F. Maienschein, R. Peelle, W. Zobel, and T. Love, "Gamma rays associated with fission," Oak Ridge National Laboratory, Tech. Rep., oct 1958.

[6] S. Oberstedt, R. Billnert, A. Gatera, W. Geerts, P. Halipré, F.-J. Hambsch, M. Lebois, A. Oberstedt, P. Marini, M. Vidali, and J. Wilson, "Prompt fission $\gamma$-ray spectra characteristics - a first summary," Physics Procedia, vol. 64, pp. 83-90, 2015.

[7] T. Kaltiaisenaho, "Implementing a photon physics model in serpent 2; fotonifysiikkamallin kehittäminen serpent 2-koodiin," Master's thesis, Aalto University, 2016.

[8] M. C. White, "Development and implementation of photonuclear crosssection data for mutually coupled neutron-photon transport calculations in the monte carlo n-particle (MCNP) radiation transport code," Los Alamos National Lab. (LANL), Tech. Rep., jul 2000.

[9] O. Pakari, V. Lamirand, B. Vandereydt, F. Vitullo, M. Hursin, C. Kong, and A. Pautz, "Design and simulation of gamma spectrometry experiments in the CROCUS reactor," in ANIMMA, Portoroz, Slovenia, 2019.

[10] Y. Nakashima, S. Minato, M. Kawano, T. Tsujimoto, and K. Katsurayama, "Gamma-ray energy spectra observed around a nuclear reactor," Journal of Radiation Research, vol. 12, no. 3-4, pp. 138-147, 1971.

[11] D. Fourmentel, P. Filliatre, J. Villard, A. Lyoussi, C. Reynard-Carette, and H. Carcreff, "Measurement of photon flux with a miniature gas ionization chamber in a material testing reactor," Nuclear Instruments and Methods in Physics Research Section A: Accelerators, Spectrometers, Detectors and Associated Equipment, vol. 724, pp. 76-82, oct 2013.

[12] G. Žerovnik, T. Kaiba, V. Radulović, A. Jazbec, S. Rupnik, L. Barbot, D. Fourmentel, and L. Snoj, "Validation of the neutron and gamma fields in the JSI TRIGA reactor using in-core fission and ionization chambers," Applied Radiation and Isotopes, vol. 96, pp. 27-35, feb 2015.

[13] K. Ambrožič, A. Gruel, V. Radulović, M. L. Guillou, P. Blaise, C. Destouches, and L. Snoj, "Delayed gamma determination at the JSI TRIGA reactor by synchronous measurements with fission and ionization chambers," Nuclear Instruments and Methods in Physics Research Section A: Accelerators, Spectrometers, Detectors and Associated Equipment, vol. 911, pp. 94-103, dec 2018.

[14] D. Fourmentel, V. Radulovic, L. Barbot, J.-F. Villard, G. Zerovnik, L. Snoj, M. Tarchalski, K. Pytel, and F. Malouch, "Delayed gamma measurements in different nuclear research reactors bringing out the importance of the delayed contribution in gamma flux calculations," in 2015 4th International Conference on Advancements in Nuclear Instrumentation Measurement Methods and their Applications (ANIMMA). IEEE, apr 2015.

[15] K. Ambrožič, V. Radulović, L. Snoj, A. Gruel, M. L. Guillou, P. Blaise, C. Destouches, and L. Barbot, "Characterization of gamma field in the JSI TRIGA reactor," EPJ Web of Conferences, vol. 170, p. 04001, 2018.

[16] M. Hursin, C. Weiss, P. Frajtag, V. Lamirand, G. Perret, P. Kavrigin, A. Pautz, and E. Griesmayer, "Testing of a sCVD diamond detection system in the CROCUS reactor," The European Physical Journal A, vol. 54, no. 5, may 2018.

[17] V. Lamirand, M. Hursin, G. Perret, P. Frajtag, O. Pakari, and A. Pautz, "Future experimental programmes in the CROCUS reactor," Conference proceedings of RRFM/IGORR 2016, 2016.

[18] O. Pakari, V. Lamirand, G. Perret, P. Frajtag, and A. Pautz, "Kinetic parameter measurements in the crocus reactor using current mode instrumentation," IEEE Transactions on Nuclear Science, 2018. 
[19] O. Pakari, V. Lamirand, G. Perret, L. Braun, P. Frajtag, and A. Pautz, "Current mode neutron noise measurements in the zero power reactor CROCUS,' in EPJ Web of Conferences, vol. 170. EDP Sciences, 2018, p. 04017.

[20] U. Kasemeyer, R. Früh, J. M. Paratte, and R. Chawla, "Benchmark on kinetic parameters in the CROCUS reactor," International Reactor Physics Experiments Handbook (IRPhE), no. 4440, OECD, 2007.

[21] J. Paratte, R. Früh, U. Kasemeyer, M. Kalugin, W. Timm, and R. Chawla, "A benchmark on the calculation of kinetic parameters based on reactivity effect experiments in the CROCUS reactor," Annals of Nuclear Energy, vol. 33, no. 8, pp. 739-748, 2006.

[22] V. Lamirand, P. Frajtag, D. Godat, O. Pakari, A. Laureau, A. Rais, M. Hursin, G. Hursin, C. Fiorina, and A. Pautz, "The colibri experimental program in the crocus reactor: characterization of the fuel rods oscillator,' in EPJ Web of Conferences, vol. 225. EDP Sciences, 2020, p. 04020 .

[23] V. Lamirand, A. Rais, O. Pakari, S. Hübner, C. Lange, J. Pohlus, U. Paquee, C. Pohl, M. Hursin, A. Laureau, P. Frajtag, D. Godat, G. Perret, C. Fiorina, and A. Pautz, "Experimental results of the first COLIBRI neutron noise campaign in the CROCUS reactor for the european project CORTEX," in Submitted to PHYSOR 2020, 2020.

[24] V. Lamirand, A. Rais, S. Hübner, C. Lange, J. Pohlus, U. Paquee, C. Pohl, O. Pakari, P. Frajtag, D. Godat, M. Hursin, A. Laureau, G. Perret, C. Fiorina, and A. Pautz, "Neutron noise experiments in the AKR-2 and CROCUS reactors for the european project CORTEX," EPJ Web of Conferences, vol. 225, p. 04023, 2020.

[25] V. Lamirand, A. Rais, O. Pakari, M. Hursin, A. Laureau, J. Pohlus, U. Paquee, C. Pohl, S. Hübner, C. Lange, P. Frajtag, D. Godat, G. Perret, C. Fiorina, and A. Pautz, "ANALYSIS OF THE FIRST COLIBRI FUEL RODS OSCILLATION CAMPAIGN IN THE CROCUS REACTOR FOR THE EUROPEAN PROJECT CORTEX," EPJ Web of Conferences, vol. 247, p. 21010, 2021.

[26] "Scionix holland, mechanical, optical and scintillation properties, https://scionix.nl/scintillation-crystals/\#tab-id-4," 2019.

[27] DSA-LX, Digital signal analyzer, https://www.mirion.com/products/dsalx-digital-signal-analyzer.

[28] O. Pakari, V. Lamirand, T. Mager, P. Frajtag, and A. Pautz, "Delayed gamma fraction determination in the zero power reactor CROCUS," EPJ $N, 2021$.

[29] O. Pakari et al., "High accuracy measurement of the prompt decay constant in CROCUS using gamma noise and bootstrapped uncertainties," Submitted to Annals of Nuclear Energy, 2021.

[30] R. Billnert, E. Andreotti, F.-J. Hambsch, M. Hult, J. Karlsson, G. Marissens, A. Oberstedt, and S. Oberstedt, "Novel scintillation detectors for prompt fission $\gamma$-ray measurements," Physics Procedia, vol. 31, pp. 2934, 2012.

[31] K. Shah, J. Glodo, W. Higgins, E. van Loef, W. Moses, S. Derenzo, and M. Weber, "CeBr/sub 3/ scintillators for gamma-ray spectroscopy," in IEEE Symposium Conference Record Nuclear Science. IEEE, 2004.

[32] W. Higgins, A. Churilov, E. van Loef, J. Glodo, M. Squillante, and K. Shah, "Crystal growth of large diameter LaBr3:ce and CeBr3," Journal of Crystal Growth, vol. 310, no. 7-9, pp. 2085-2089, apr 2008.

[33] O. Pakari, "Experimental and numerical study of stochastic branching noise in nuclear reactors," Ph.D. dissertation, École polytechnique fédérale de Lausanne (EPFL), 2020.

[34] M. J. Weber and R. R. Monchamp, "Luminescence of Bi4 Ge3 O12 : Spectral and decay properties," Journal of Applied Physics, vol. 44, no. 12 , pp. 5495-5499, dec 1973.

[35] O. H. Nestor and C. Y. Huang, "Bismuth germanate: A high-z gammaray and charged particle detector," IEEE Transactions on Nuclear Science, vol. 22, no. 1, pp. 68-71, 1975.

[36] S. E. Brunner and D. R. Schaart, "BGO as a hybrid scintillator / cherenkov radiator for cost-effective time-of-flight PET," Physics in Medicine and Biology, vol. 62, no. 11, pp. 4421-4439, may 2017.

[37] M. Gierlik, T. Batsch, M. Moszynski, T. Szczesniak, D. Wolski, W. Klamra, B. Perot, and G. Perret, "Comparative study of large $\mathrm{NaI}(\mathrm{tl})$ and BGO scintillators for the EURopean illicit TRAfficking countermeasures kit project," IEEE Transactions on Nuclear Science, vol. 53, no. 3, pp. 1737-1743, jun 2006.

[38] V. Lamirand, A. Laureau, O. Pakari, P. Frajtag, and A. Pautz, "Power calibration methodology at the CROCUS reactor," EPJ Web of Conferences, vol. 225, p. 04022, 2020.

[39] G. Perret, "Tm-41-14-02 rev. 1: Decay constant and delayed neutron fraction measurements in crocus," ERP, LRS, Paul Scherrer Institut, Tech. Rep., 102014.

[40] G. Knoll, Radiation detection and measurement. John Wiley \& Sons, 2010 .
[41] J. Hubbell, "Photon mass attenuation and energy-absorption coefficients," The International Journal of Applied Radiation and Isotopes, vol. 33, no. 11, pp. 1269-1290, 1982.

[42] O. V. Pakari, "Development of current and fast neutron noise measurements in CROCUS," Master's thesis, École polytechnique fédérale de Lausanne (EPFL), 2016. [Online]. Available: http://infoscience.epfl.ch/record/253177

[43] Database of prompt gamma rays from slow neutron capture for elemental analysis. Vienna: International Atomic Energy Agency, 2007.

[44] M.-M. Bé, V. Chisté, C. Dulieu, M. Kellett, X. Mougeot, A. Arinc, V. Chechev, N. Kuzmenko, T. Kibédi, A. Luca, and A. Nichols, Table of Radionuclides, ser. Monographie BIPM-5. Pavillon de Breteuil, F92310 Sèvres, France: Bureau International des Poids et Mesures, 2016, vol. 8.

[45] D. E. Alburger and G. A. P. Engelbertink, "Half-lives ofBe11,c15,n16,o19, andA128," Physical Review C, vol. 2, no. 5, pp. 1594-1596, nov 1970.

[46] H. H. Schmidt, P. Hungerford, H. Daniel, T. von Egidy, S. A. Kerr, R. Brissot, G. Barreau, H. G. Börner, C. Hofmeyr, and K. P. Lieb, "Levels and gamma energies of A128 studied by thermal neutron capture," Physical Review C, vol. 25, no. 6, pp. 2888-2901, jun 1982.

[47] K. Shozugawa, N. Nogawa, and M. Matsuo, "Deposition of fission and activation products after the fukushima dai-ichi nuclear power plant accident," Environmental Pollution, vol. 163, pp. 243-247, apr 2012.

[48] W. Kim, M. Hursin, A. Pautz, L. Vincent, F. Pavel, and D. Lee, "Determination of the activity inventory and associated uncertainty quantification for the CROCUS zero power research reactor," Annals of Nuclear Energy, vol. 136, p. 107034, feb 2020.

[49] J. W. Durkee, M. R. James, G. W. McKinney, L. S. Waters, and T. Goorley, "The MCNP6 delayed-particle feature," Nuclear Technology, vol. 180, no. 3, pp. 336-354, dec 2012.

[50] V. Lamirand, O. Pakari, F. Vitullo, K. Ambrožič, D. Godat, P. Frajtag, and A. Pautz, "Local and high distance neutron and gamma measurements of fuel rods oscillation experiments," ANIMMA 2021, 2021.

[51] O. Pakari et al., "High distance measurements of the prompt decay constant in the CROCUS reactor using gamma noise," Submitted to Annals of Nuclear Energy, 2021. 